Poland's growing share in the immense task of exploring the Cosmos, in which our Great Compatriot was a pioneer.

We are glad this session of world astronomers will give them an opportunity to acquaint themselves with the achievements of our science, with the gains of post-war Poland, with the presence and inspiring strength of Copernican traditions in our country.

I wish all members of the extraordinary session of the General Assembly of the International Astronomical Union that your debates-thanks to the presentation of the latest research achievements, the exchange of opinions and creative inspirations-make a valuable contribution to the progress of cognitive thought and to the pool of scientific knowledge, to strengthening peace and cooperation among nations, to enriching Copernican traditions which unite us all and make us build human science in a human world.'

\title{
ADDRESS BY THE PRESIDENT OF THE POLISH ACADEMY OF SCIENCES, PROF. W. TRZEBIATOWSKI
}

'Mr Prime Minister, Mr President of the International Astronomical Union, Distinguished Guests,

The Extraordinary Gencral Assembly of the International Astronomical Union which we are opening today is an occurrence of exceptional importance. In its international aspect, it marks the culminating point of this year's Copernican observances. For the participation in this session of astronomers from all parts of the world we accept, among other things, as a tribute paid to our Great Compatriot, the founder of modern astronomy, the bedrock of the present-day outlook on the world.

The session is at the same time a scientific gathering with an extensive working programme. At the symposia starting tomorrow, all the latest astronomic problems will be discussed. We are glad the symposia will be held in Poland's three academic centres: Warsaw, Toruń and Cracow, and will have active participation of Polish astronomers and physicists. That will provide a particularly important and valuable opportunity to strengthen the existing and establish new personal ties between Polish and foreign scholars.

Characteristically, two of the mentioned symposia, being a part of this Extraordinary General Assembly, will be devoted to subjects related to physics and astrophysics, while the third one will be dealing with exploratory problems of cosmic physics. That programme demonstrates the important role contemporary astronomy plays in the family of natural sciences, its growing correlation with physics and ever stronger feedback with all those branches of science and technology involved in the exploration and peaceful use of outer space. Aware of this, the Polish Academy of Sciences accords astronomy a prominent place in its long-range plans for the development of scientific agencies and the research conducted thereby. We wish that Polish astronomy make a contribution to world science that would be proportional to our ambitions, that it continue the valuable Copernican traditions.

On behalf of the Polish Academy of Sciences, and on my own behalf, I would like to most sincerely welcome all members of the Extraordinary General Assembly of the International Astronomical Union. I wish you a successful debate, inspiring scientific impressions, and a pleasant stay in this country.'

\section{ADDRESS BY THE CHAIRMAN OF THE WARSAW CITY COUNCIL, MR. MAJEWSKI}

'Distinguished Guests,

On behalf of Warsaw inhabitants and authorities, I have the honour to welcome in the capital of People's Poland members of the Extraordinary General Assembly of the International Astronomical Union. I wish to express my great satisfaction that we can play host to so many outstanding representatives of such a magnificent and universal domain of science as astronomy is and that your presence in our town is a sign of appreciation for the contribution to the history of human thought made by a great Pole, Mikolaj Kopernik. 
Warsaw, which is your host town, is a city with a magnificent and at the same time dramatic history. Four days ago we celebrated the thirty-fourth anniversary of the outbreak of World War II. First bombs in that war fell on our city. But it was only the beginning of a tragedy. Over five years later, the final balance-sheet of destruction was horrifying: out of every ten buildings of the pre-war Warsaw nine had been destroyed, burned or blown up. The city centre, objects of culture and science, relics of the past, industry had all been completely destroyed. Over 800 thousand Warsaw inhabitants died under Nazi terror.

I mention this to emphasize the determination to survive, so characteristic of Warsaw inhabitants. Today, Warsaw reconstructed with the effort of the whole nation, is again a properly functioning, developing city. I hope that despite the extensive programme of your Assembly you find time to see that for your own. At the same time, however, I would like to turn your attention to Warsaw's achievements in the domain well-familiar to you, in science.

The traditions of our city in this domain are by no means insignificant. I think, for example, the traditions of the Warsaw mathematical school are not unknown to you. In the post-war period efforts at reconstruction of residential quarters and industrial establishments, relics of the past and monuments, were accompanied by a consistent strive to revive and subsequently develop the scientific base: higher schools, laboratories, and research centres. Today, nearly $30 \%$ of the country's scientific potential is concentrated in Warsaw and the achievements of our scientists, at least in proportion to the ratio of this concentration, are notable in more than one domain.

Astronomical traditions of Warsaw, which date back to over a hundred and fifty years ago, to the date of establishment of the first observatory, are being continued in a modern way. Construction work will begin shortly on the Copernican Centre, the first element of a big concentration of intellectual and research potential, known as the Warsaw Scientific Complex. I am very pleased to say that the Copernican Centre which is to serve the exchange of astronomical thought regardless of frontiers and social systems, will be constructed under international cooperation, the same nobleminded and peaceful cooperation which has brought you here to Warsaw.

I wish you, Distinguished Guests, fruitful debates and a pleasant stay in our city.'

\section{ADDRESS BY THE CHAIRMAN OF THE NATIONAL COMMITTEE FOR THE IAU,} PROFESSOR W. IWANOWSKA

'Mister Chairman of the Council of Ministers, Mister President of the International Astronomical Union, Dear Guests,

The reason why our National Committee for Astronomy was so anxious to have a Meeting of the International Astronomical Union in our country this year, was the 500th Anniversary of the birth of Nicolaus Copernicus. One could think that celebrating anniversaries of great men of science is a nice custom, but-in the present very busy life-it is something like a waste of time. One might also think that studying the contributions of great men to the development of science belongs to the domain of the history of science.

All this being true, we think - however-that it is useful for scientists, once per generation, to have a look back on the way already passed, in order to get a perspective for the future plans. The span of time between the Copernican "Revolutions" and the present day revolution in science and technology offers such an opportunity; I should say-an excellent opportunity, because both ends of this span are exceptional and unforeseen. The work of Copernicus was a starting point of a continuous, exponential development of modern science and modern technology, whereas the present day acceleration of their growth begins to raise problems to the mankind, how to manage and organize research work and its applications.

In both these moments astronomy played and plays a leading part. In fact, astronomy is a leading science among natural sciences, since it stores in the depths of the Universe the greatest resources of the Unknown. It was not really an accident that the development of modern science started in astronomy. To find the fundamental laws of mechanics, the law of universal gravitation, a laboratory at least as big as the planetary system was necessary and this laboratory should first be brought into 